DRAFT VERSION JunE 22, 2020

Preprint typeset using $\mathrm{LAT}_{\mathrm{E}} \mathrm{X}$ style AASTeX6 v. 1.0

\title{
INFERENCES ABOUT THE MAGNETIC FIELD STRUCTURE OF A CME WITH BOTH IN SITU AND FARADAY ROTATION CONSTRAINTS
}

\author{
Brian E. Wood ${ }^{1}$, Samuel Tun-Beltran ${ }^{1}$, Jason E. Kooi ${ }^{2}$, Emil J. Polisensky ${ }^{2}$, Teresa Nieves-Chinchilla ${ }^{3}$ \\ ${ }^{1}$ Naval Research Laboratory, Space Science Division, Washington, DC 20375, USA; brian.wood@nrl.navy.mil \\ ${ }^{2}$ Naval Research Laboratory, Remote Sensing Division, Washington, DC 20375, USA \\ ${ }^{3}$ Heliophysics Science Division, NASA Goddard Space Flight Center, Greenbelt, MD 20771, USA
}

\begin{abstract}
On 2012 August 2, two CMEs (CME-1 and CME-2) erupted from the west limb of the Sun as viewed from Earth, and were observed in images from the white light coronagraphs on the $S O H O$ and STEREO spacecraft. These events were also observed by the Very Large Array (VLA), which was monitoring the Sun at radio wavelengths, allowing time-dependent Faraday rotation observations to be made of both events. We use the white-light imaging and radio data to model the 3-D field geometry of both CMEs, assuming a magnetic flux rope geometry. For CME-2, we also consider 1 au in situ field measurements in the analysis, as this CME hits STEREO-A on August 6, making this the first CME with observational constraints from stereoscopic coronal imaging, radio Faraday rotation, and in situ plasma measurements combined. The imaging and in situ observations of CME-2 provide two clear predictions for the radio data; namely that VLA should observe positive rotation measures $(\mathrm{RMs})$ when the radio line of sight first encounters the CME, and that the sign should reverse to negative within a couple hours. The initial positive RMs are in fact observed. The expected sign reversal is not, but the VLA data unfortunately end too soon to be sure of the significance of this discrepancy. We interpret an RM increase prior to the expected occultation time of the CME as a signature of a sheath region of deflected field ahead of the CME itself.
\end{abstract}

Keywords: Sun: coronal mass ejections (CMEs) — solar wind — interplanetary medium

\section{INTRODUCTION}

Coronal mass ejections (CMEs) are among the most dramatic of solar eruptive phenomena, which can have significant geomagnetic effects when directed toward Earth. Assessing the structure of CMEs and predicting their geoeffectiveness has therefore been a central goal of heliophysics. The primary observational diagnostics of CMEs are white-light images of the transients. The most prodigious source of these observations over the past two decades is the Large Angle Spectrometric COronagraph (LASCO) instrument on board the SOlar and Heliospheric Observatory (SOHO). From SOHO's vantage point at the Sun-Earth L1 Lagrangian point, the LASCO C2 and C3 coronagraphs have been monitoring the solar corona within $30 \mathrm{R}_{\odot}$ of the Sun since 1996 (e.g., Yashiro et al. 2004; Gopalswamy et al. 2009; Robbrecht et al. 2009).

However, interpreting images of CMEs can be difficult, due to the problem of inferring three-dimensional structure from two-dimensional images. Although CMEs are often described in terms of a three-part structure, with a bright core near the back end of a dark cavity surrounded by a bright loop (Illing \& Hundhausen 1985; Gibson \& Low 1998), their appearance can vary tremendously from event to event. A crucial advance in CME studies has been the advent of stereoscopic imaging of CMEs, particularly using observations from the Solar TErrestrial RElations Observatory (STEREO) mission, with twin spacecraft (STEREO-A and STEREO-B) monitoring the solar corona and interplanetary space from two separate vantage points 1 au from the Sun. Analyses of these data have provided support for the magnetic flux rope (MFR) paradigm of CME structure, where CMEs are assumed to be long tubes permeated by a helical magnetic field, with the legs of the tubes stretching back toward the Sun (Thernisien et al. 2009; Kilpua et al. 2012; Vourlidas et al. 2014; Wood et al. 2017; Möstl et al. 2018). Stereoscopic analysis of CMEs has recently been extended to include observations from close to the Sun by the Parker Solar Probe (Wood et al. 2020).

Stereoscopic imaging may provide excellent constraints on a CME's 3-D shape and kinematics, if the viewing geometry is advantageous, but this by itself does not elucidate the magnetic structure of the event. Images only show 
mass within the CME, which may not relate well to the magnetic field that provides the framework of the CME. Furthermore, it is the magnetic field strength and orientation that largely determines whether an Earth-directed CME will be geoeffective or not. Thus, observational constraints on magnetic fields within CMEs are crucial for studies of CME structure.

The most ubiquitous source of field measurements of CMEs are magnetometers that are on board many heliophysics missions, including STEREO, Wind, and Advanced Composition Explorer (ACE); the latter two operating near Earth at L1. Like the imaging data, in situ plasma and field measurements also provide support for the MFR paradigm of CME structure, with many interplanetary CMEs (i.e., ICMEs) characterized by a region of low plasma $\beta$ and strong, rotating magnetic field, consistent with the MFR picture (Burlaga et al. 1981; Marubashi 1986; Burlaga 1988; Bothmer \& Schwenn 1998). The spacecraft field measurements suffer from one obvious drawback, and that is that they only provide measurements for a single track through the CME structure. Techniques have been developed to extrapolate from that track to a full 3-D MFR structure, but such extrapolations require simplifying assumptions about the physics and geometry of the MFR structure (e.g., Lepping et al. 1990, 2011, 2015; Hu \& Sonnerup 2001; Vandas \& Romashets 2003; Al-Haddad et al. 2013; Nieves-Chinchilla et al. 2016, 2018).

Radio observations potentially provide an alternative source of information about CME magnetic field structure, specifically by measuring the Faraday rotation induced by a CME passing in front of a background radio source. There is a long history of using Faraday rotation to study coronal plasma (e.g., Bird et al. 1980; Sakurai \& Spangler 1994; Ingleby et al. 2007; Ord et al. 2007; Kooi et al. 2014), but measuring CMEs is naturally harder due to their unpredictable and transient nature. The first serendipitous detections involved radio signals from Pioneer 9 (Levy et al. 1969). A more systematic effort specifically designed to detect CMEs was made involving Helios, resulting in five detections (Bird et al. 1985). More recently, there has been a study utilizing the signal from the MESSENGER spacecraft (Jensen et al. 2018). Detections using astrophysical background sources are few. Howard et al. (2016) failed to detect a rotation measure signal in Very Large Array (VLA) observations of a pulsar that was occulted by a CME, although limits were placed on the CME's field strength. However, (Kooi et al. 2017) reported successful VLA detections of three CMEs on 2012 August 2, and the Low Frequency Array (LOFAR) detected a rotation measure signal even farther from the Sun for an event on 2014 August 13 (Bisi et al. 2016).

We have recently recognized that one of the events observed by VLA on 2012 August 2 was directed right at STEREO-A, and hit the spacecraft on 2012 August 6, where it was observed by the spacecraft's particle and field detectors. The STEREO viewing geometry of the event was also excellent, at least while near the Sun, providing the necessary data for an accurate morphological reconstruction. All this makes this event truly unique, as being the only CME with observational constraints from both stereoscopic imaging and Faraday rotation observations near the Sun, and also in situ plasma measurements at 1 au. This is therefore the ideal event for exploring CME field structure, and we here present our study of the CME considering all of the observational constraints as listed.

The analysis begins in Section 2 with a description of our morphological and kinematic measurements based on the stereoscopic imaging constraints. The morphological reconstruction is based on the MFR paradigm for CME structure. There are actually two distinct CMEs that erupt within two hours of each other off the west limb of the Sun, as viewed from Earth. Although we focus mostly on the second CME that ultimately hits STEREO-A, the first CME is analyzed as well, as radio Faraday rotation constraints are available for both CMEs using two separate background radio sources. In Section 3, we present STEREO-A's in situ plasma and field measurements of the second CME, taken on 2012 August 6 when that CME hits the spacecraft. These data provide the basis by which we insert a physically realistic field structure into the MFR morphology inferred from the imaging. This analysis utilizes the new MFR model of Nieves-Chinchilla et al. (2018, hereafter TNC18). In Section 4, we focus on the radio Faraday rotation data, with a primary goal of determining whether the MFR reconstruction of the CME morphology and field structure from the imaging and 1 au in situ data is consistent with the radio constraints. Finally, in Section 5 we summarize our results.

\section{RECONSTRUCTION OF CME MORPHOLOGY FROM STEREOSCOPIC IMAGING}

The coronagraphic observations of the CMEs on 2012 August 2 were first described by Kooi et al. (2017). These include observations from SOHO/LASCO's C2 and C3 coronagraphs, covering plane-of-sky distances from Sun-center of 1.5-6 $\mathrm{R}_{\odot}$ and 3.7-30 $\mathrm{R}_{\odot}$, respectively (Brueckner et al. 1995); and also by the COR1 and COR2 coronagraphs on board the twin STEREO spacecraft, covering distances of 1.4-4.0 $\mathrm{R}_{\odot}$ and 2.5-15.6 $\mathrm{R}_{\odot}$, respectively, which are constitutents of STEREO's Sun-Earth Connection Coronal and Heliospheric Investigation (SECCHI) imaging package (Howard et al. 2008). Utilizing the CME identifiers of Kooi et al. (2017), CME-1 was first seen at UT 13:25 by LASCO/C2, directed in a roughly southwesterly direction. This CME is followed shortly thereafter by CME-2 at UT 

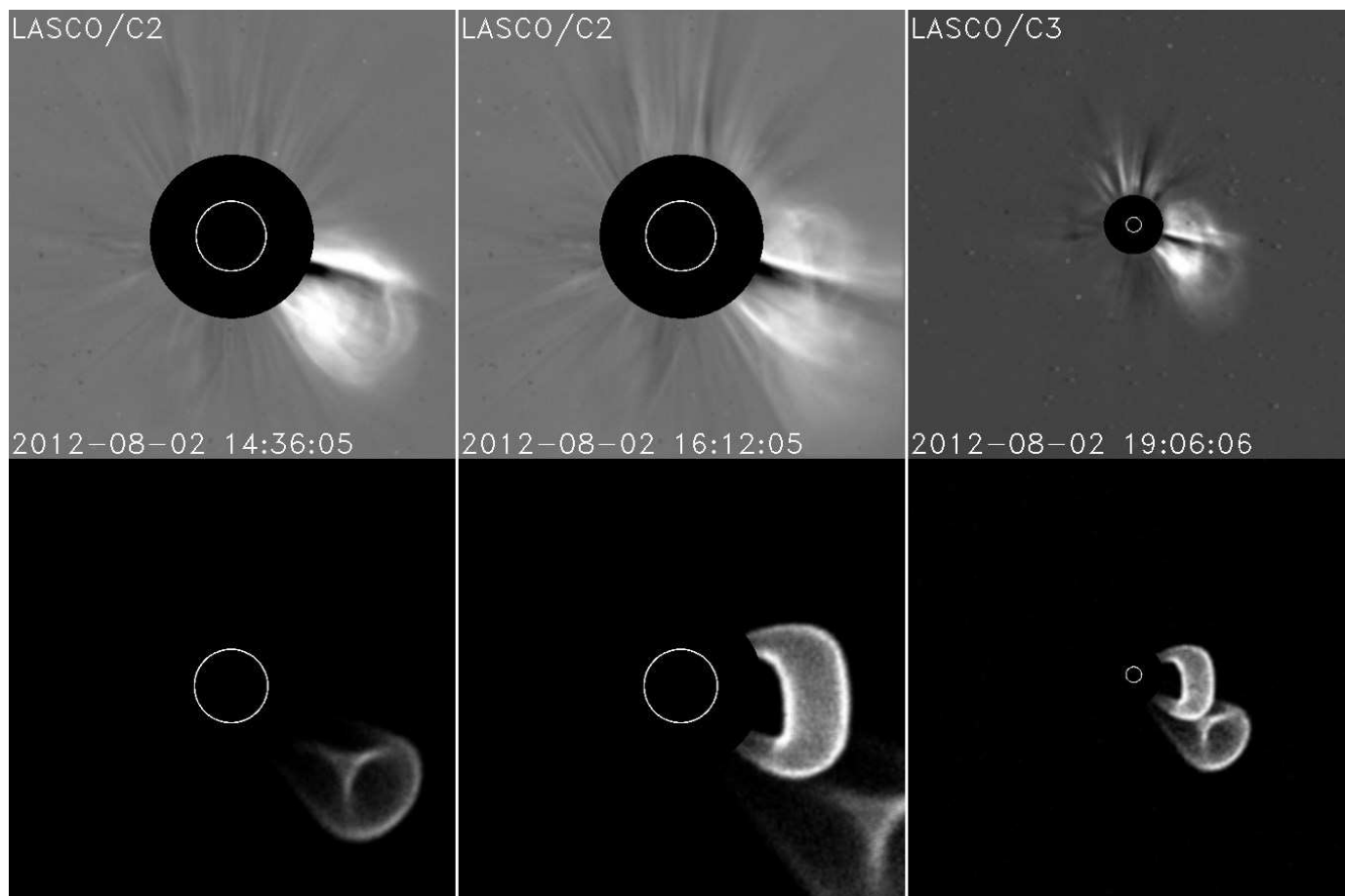

Figure 1. Images of the 2012 August 2 CMEs from SOHO/LASCO's C2 and C3 corongraphs. The first C2 image shows CME-1, the second CME-2, and the third image shows the overlapping CMEs in the C3 FOV. Synthetic images of the event are shown below the real images, based on the 3-D reconstruction shown in Figure 4. A movie version of this figure is available online.

14:48, which overlaps CME-1 but with a trajectory more directly to the west. This is shown explicitly in the first two panels of Figure 1, with the third panel showing the overlapping CMEs in the field of view (FOV) of LASCO's C3 coronagraph. Kooi et al. (2017) discuss a narrow third CME, CME-3, directed to the northwest at UT 16:36, which we do not consider in this article.

Figure 2 shows a sequence of four synchronised COR1 and COR2 images of the two CMEs from the two STEREO spacecraft. Interpretation of the images requires knowledge of the viewing geometry. This is shown explicitly in Figure 3(a), which illustrates the positions of the various observing locations in a heliocentric Earth ecliptic (HEE) coordinate system, with the x-axis pointed toward Earth and the z-axis pointed toward ecliptic north. The COR2-B image at UT 18:39 greatly resembles the LASCO/C3 image at UT 19:06 in Figure 1, but with the CMEs on the east side of the Sun instead of the west. This implies that the CMEs are directed roughly halfway between $S O H O /$ LASCO and STEREO-B, which places their trajectories roughly toward the longitude of STEREO-A (see Figure 3a). The $S T E R E O-A$ images provide support for this conclusion. From $S T E R E O-A$ 's perspective, CME-1 seems directed close to due south, but slightly to the east. Finding CME-2 in the STEREO-A images is trickier. In the COR2-A image at UT 18:39 in Figure 2, CME-2 is apparent as a faint elliptical halo CME surrounding the Sun. This is more apparent in the movie version of the figure. (Movie versions of both Figures 1 and 2 are available in the online version of the article.) The halo appearance of the CME indicates that the CME is directed right at STEREO-A, a trajectory direction supported by the combined appearance of the CME in STEREO-B and LASCO. Furthermore, CME-2 in fact hits $S T E R E O-A$ four days later on August 6, as will be discussed in Section 3.

We measure the kinematics of the two CMEs using the STEREO-B images, with results shown in Figure 3(b). We first measure the elongation angle, $\epsilon$, of each CME's leading edge from Sun-center as a function of time. Following past practice (e.g., Wood et al. 2010, 2017), these angles are converted to physical distances using the prescription of Lugaz et al. (2009),

$$
r=\frac{2 d \sin \epsilon}{1+\sin (\epsilon+\phi)}
$$

where $d$ is the distance from the observer (STEREO-B in this case) to the Sun and $\phi$ is the angle between the CME trajectory and the observer's line-of-sight (LOS) to the Sun. This equation assumes the CME can be approximated as a sphere centered halfway between the Sun and the CME's leading edge. The central trajectory and $\phi$ values for the two CMEs are ultimately inferred from the morphological analysis described below, with the central longitude of those trajectories shown explicitly in Figure 3(a). The CME distances are fitted with a simple two-phase kinematic model 


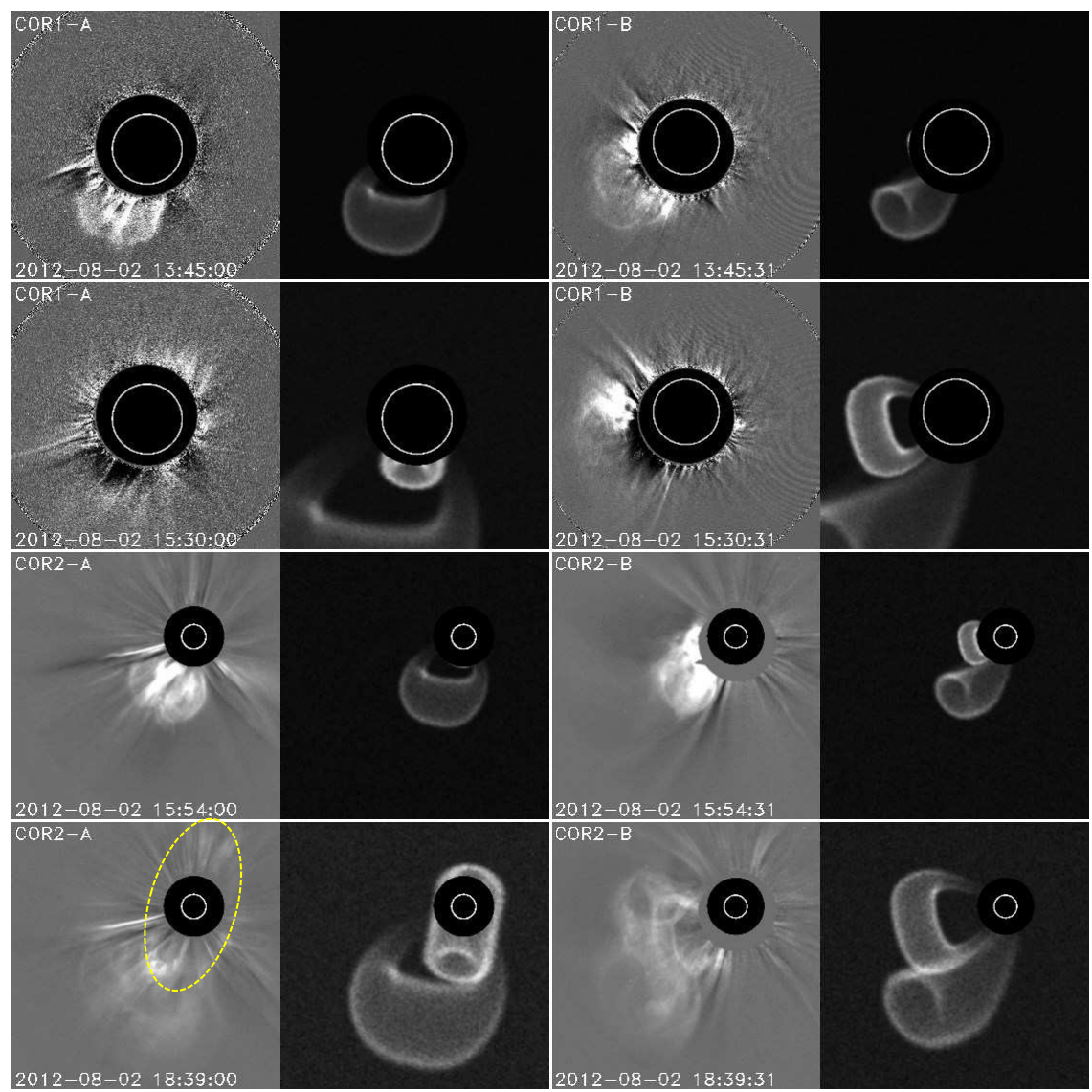

Figure 2. A sequence of four synchronised images of the 2012 August 2 CMEs from the COR1 and COR2 coronagraphs on STEREO-A (left) and STEREO-B (right). The first COR1 image shows CME-1, and the second CME-2, which follows right behind CME-1. The third image shows primarily CME-1 in the COR2 FOV, and the final image shows the overlapping CMEs in COR2. For clarity, a yellow dashed line is used in the final COR2-A image to outline CME-2's location. Synthetic images of the event are shown to the right of the real images, based on the 3-D reconstruction shown in Figure 4 . A movie version of this figure is available online.

assuming an initial phase of constant acceleration followed by a coast phase of constant velocity, with the results shown in Figure 3(b). Neither CME is very fast, with CME-1 reaching a peak speed of $506 \mathrm{~km} \mathrm{~s}^{-1}$, and CME-2 reaching 461 $\mathrm{km} \mathrm{s}^{-1}$.

Both STEREO- $A$ and - $B$ possess heliospheric imagers that are often able to track CMEs into interplanetary space, potentially all the way to $1 \mathrm{au}$ (e.g., Howard et al. 2008; Eyles et al. 2009; Wood et al. 2017). However, the trajectories of the two 2012 August 2 CMEs do not place them in the fields of view of the heliospheric imagers, which are pointed at the Sun-Earth line. The central longitude of the CME-1 trajectory shown in Figure 3(a) suggests that it might be in the heliospheric imager FOV for STEREO-A, but in reality the CME trajectory latitude is too far to the south. The lack of heliospheric imager constraints means that our kinematic models of the two CMEs are based only on the coronagraphic observations close to the Sun. Nevertheless, in Section 3 we will show that the kinematic model of CME-2 in Figure 3(b) assuming that the CME maintains the $461 \mathrm{~km} \mathrm{~s}^{-1}$ speed all the way to $S T E R E O-A$ predicts the CME arrival time at STEREO-A surprisingly well. This success probably indicates that the CME speed was close enough to that of the ambient solar wind to allow the CME to reach 1 au without further acceleration or deceleration.

We use the stereoscopic imagery of each CME to reconstruct its 3-D morphology, asssuming an underlying MFR shape. For this purpose, we use a parametrized mathematical prescription for generating 3-D MFR shapes that we have used many times in the past. The prescription is described in detail by Wood \& Howard (2009), but is most extensively utilized in the STEREO CME survey of Wood et al. (2017). An assumed set of MFR parameters yields a 3-D MFR shape. This shape is then used to generate a 3-D density cube, with mass placed only on the surface of the MFR to outline the CME boundary, and not in the interior. We assume self-similar expansion for the MFR, meaning that this single density cube applies at all times, with only the axis scale changing in a manner described by the kinematic model of the CME (e.g., Figure 3b). Thus, from the 3-D density cube we can compute synthetic images of the CME for any FOV from any perspective at any time, for comparison with the actual images of the CME. The synthetic images are computed using a white light rendering routine that precisely computes the Thomson scattering 

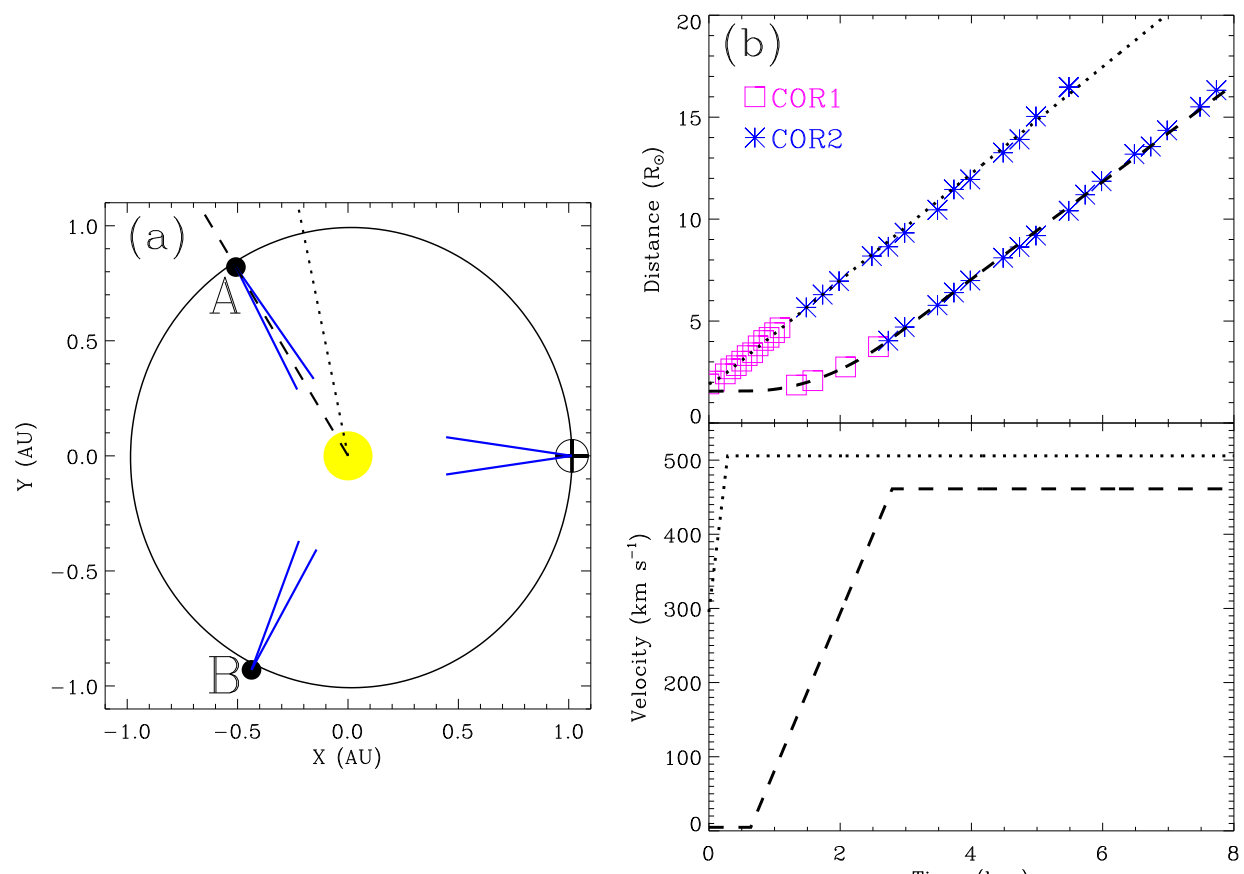

Figure 3. (a) The positions of Earth, STEREO-A, and STEREO-B in the ecliptic plane on 2012 August 5 (in HEE coordinates). From each of these perspectives the blue lines show the fields of view of SOHO/LASCO's C3 coronagraph, and the COR2-A and COR2-B coronagraphs on STEREO-A and -B, respectively. The dotted and dashed lines indicate the central trajectory longitude of CME-1 and CME-2, respectively. (b) The top panel shows distance measurements for the leading edges of CME-1 and CME-2 based on images from STEREO-B's coronagraphs (COR1-B and COR2-B). The $t=0$ point on the time axis corresponds to UT 12:55 on August 2. These data are fitted with a simple kinematic model assuming a constant acceleration phase followed by a constant velocity phase. The dotted and dashed lines are the best fits. The corresponding dotted and dashed lines in the bottom panel show the inferred velocity profiles for CME-1 and CME-2, respectively.
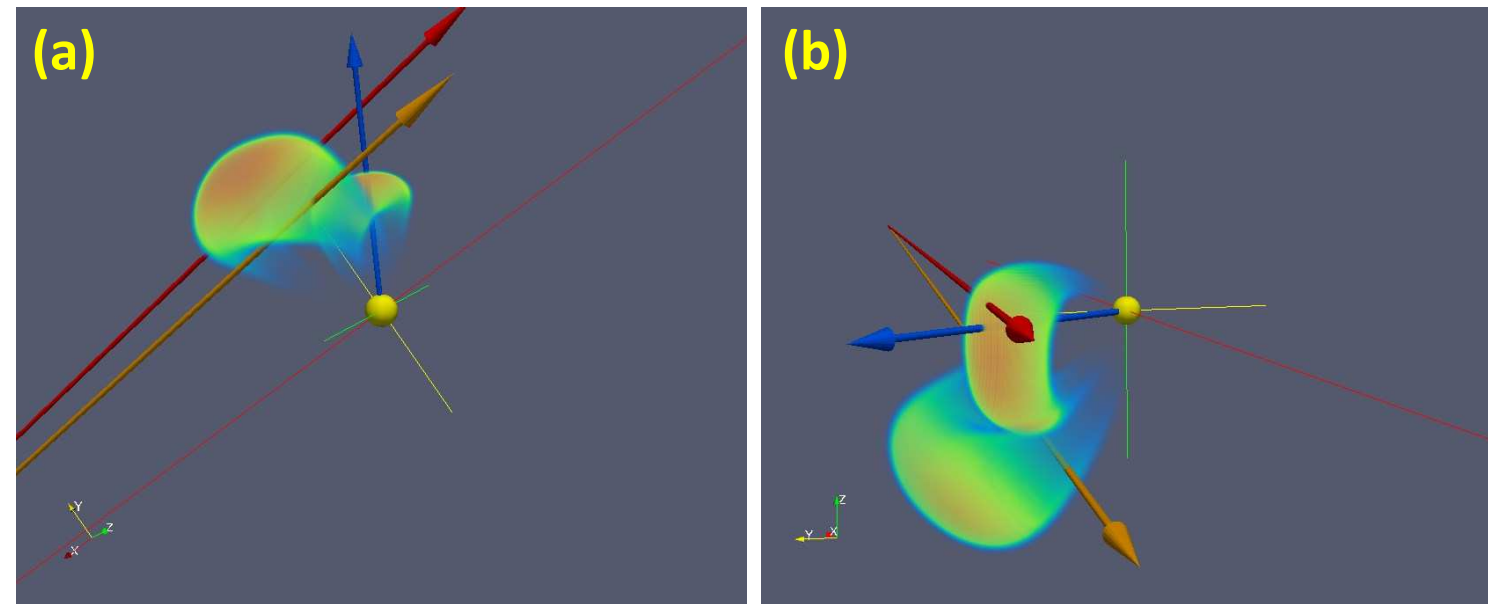

Figure 4. (a) Reconstructed 3-D MFR structures of CME-1 and CME-2, at UT 17:24, shown in HEE coordinates. CME-1 is the larger, E-W oriented MFR, and CME-2 is the smaller N-S oriented MFR. The Sun is at the origin, and is shown to scale. The blue arrow indicates the direction toward STEREO-A, which goes through CME- 2 . The red and orange arrows indicate the LOS from Earth toward two background radio sources, 0842+1835 (red) and 0843+1547 (orange), observed by VLA for Faraday rotation purposes. The 0842 LOS goes through CME-2 and the 0843 LOS samples CME-1. (b) Same as (a), but for a later time, UT 19:24.

within the density cube (Billings 1966; Thernisien et al. 2006).

Simple trial-and-error and subjective judgment are used to vary the MFR parameters and decide which parameters yield synthetic images that collectively best match the actual images. Our final inferred MFR morphologies of CME-1 and CME-2 are depicted in Figure 4, which shows the relative positions of the two CMEs at two particular times. Synthetic images of this morphological reconstruction are shown in Figures 1 and 2, for comparison with the actual images. (Movie versions of these figures in the online article provide a more comprehensive data/model comparison.) 
Table 1. Flux Rope Parameters

\begin{tabular}{clcc}
\hline \hline Parameter & \multicolumn{1}{c}{ Description } & CME-1 & CME-2 \\
\hline$\lambda_{s}(\mathrm{deg})$ & Trajectory longitude & 102 & 122 \\
$\beta_{s}(\mathrm{deg})$ & Trajectory latitude & -40 & -10 \\
$\gamma_{s}(\mathrm{deg})$ & Tilt angle of MFR & -15 & 80 \\
$\mathrm{FWHM}_{s}(\mathrm{deg})$ & Angular width & 75.8 & 77.1 \\
$\Lambda_{s}$ & Aspect ratio & 0.18 & 0.15 \\
$\eta_{s}$ & Ellipticity of MFR cross section & 1.5 & 1.7 \\
$\alpha_{s}$ & Shape parameter for leading edge & 3.0 & 5.0 \\
\hline
\end{tabular}

Table 1 lists the MFR fit parameters for CME-1 and CME-2, using the variable names from Wood et al. (2017). Briefly, $\lambda_{s}$ and $\beta_{s}$ describe the central trajectories in HEE coordinates, with the $\lambda_{s}$ directions explicitly indicated in Figure 3(a). The $\gamma_{s}$ parameter indicates the tilt angle of the MFR, with $\gamma_{s}=0^{\circ}$ corresponding to an E-W orientation parallel to the ecliptic, and $\gamma_{s}>0^{\circ}$ indicating an upward tilt of the west leg. With $\gamma_{s}=80^{\circ}$, CME-2 is oriented close to N-S. This orientation is strongly implied by the clear N-S asymmetry of the CME halo as viewed in the last COR2-A image in Figure 2, and the apparent face-on MFR appearance of CME-2 in the COR2-B and LASCO/C3 images. Getting this orientation correct is crucial for interpretation of the STEREO-A in situ observations of CME-2 in the next section. The $\mathrm{FWHM}_{s}$ parameter is the full-width-at-half-maximum angular width of the MFR. The aspect ratio, $\Lambda_{s}$, indicates the minor radius of the apex of the MFR divided by the distance of the apex from the Sun, and so is a measure of how fat the MFR is. The ellipticity of the MFR channel is described by $\eta_{s}$, which is the major radius divided by the minor radius. A value of $\eta_{s}=1$ would indicate a circular MFR cross section. Finally, the $\alpha_{s}$ parameter defines the shape of the MFR leading edge, with higher values leading to flatter leading edges.

\section{RECONSTRUCTION OF CME FIELD STRUCTURE FROM IN SITU DATA}

With the morphological and kinematic reconstruction of the 2012 August 2 CMEs complete, we now turn our attention to the in situ observations of CME-2 from STEREO-A, which we use to model the magnetic field structure of the CME. The STEREO in situ observations are made by two separate instruments: the Plasma and Suprathermal Ion Composition (PLASTIC) instrument (Galvin et al. 2008), and the In situ Measurements of Particles and CME Transients (IMPACT) package (Acuña et al. 2008; Luhmann et al. 2008). Figure 5 displays the $S T E R E O-A$ in situ observations for a 3.5 day period starting on 2012 August 5, corresponding to day-of-year (DOY) 218. The top four panels show the magnetic field measurements, both the total field strength, $B_{t o t}$, and the individual $B_{x}$, $B_{y}$, and $B_{z}$ field components in a spacecraft-centered RTN coordinate system. The bottom three panels show the proton speed, number density, and temperature.

The vertical dotted lines indicate the predicted encounter time of the CME-2 MFR based on the morphological and kinematic analysis shown in Figures 3 and 4. This corresponds nicely to a period of enhanced $B_{t o t}$ and decreased $T$, which are often interpreted as ICME signatures, leading us to conclude that this is indeed the in situ signature of CME-2. The velocity and density profiles predicted by the MFR reconstruction are explicitly shown in the velocity and density panels of Figure 5. The velocity decrease predicted by the MFR reconstruction is a consequence of the assumed self-similar expansion of the MFR, which leads to trailing parts of the MFR expanding more slowly than the leading edge. The predicted velocity profile agrees very well with the observed velocities early in the ICME period, but the observed velocities deviate to higher values at later times.

Since our 3-D reconstruction only places mass on the surface of the MFR, with a Gaussian density profile of narrow width, the predicted density signature at STEREO-A is simply two Gaussian peaks corresponding to the times when the spacecraft enters and then exits the MFR. There is a sharp density peak very near the predicted time of arrival of the ICME at STEREO-A. The predicted peak is only about 2.5 hours too late. This is a surprising degree of success for the kinematic model in Figure 3(b), considering that it is based only on coronagraph observations close to the Sun, therefore requiring almost four days of extrapolation to $S T E R E O-A$ in the absence of additional constraints from STEREO's heliospheric imagers.

Even more surprising is that the ICME encounter time looks like a plausible match to the data, corresponding roughly to the duration of enhanced $B_{t o t}$, and the duration of low $T$. We have more commonly found that MFR shapes inferred from imaging usually lead to predicted ICME encounter times at 1 au that are significantly longer 


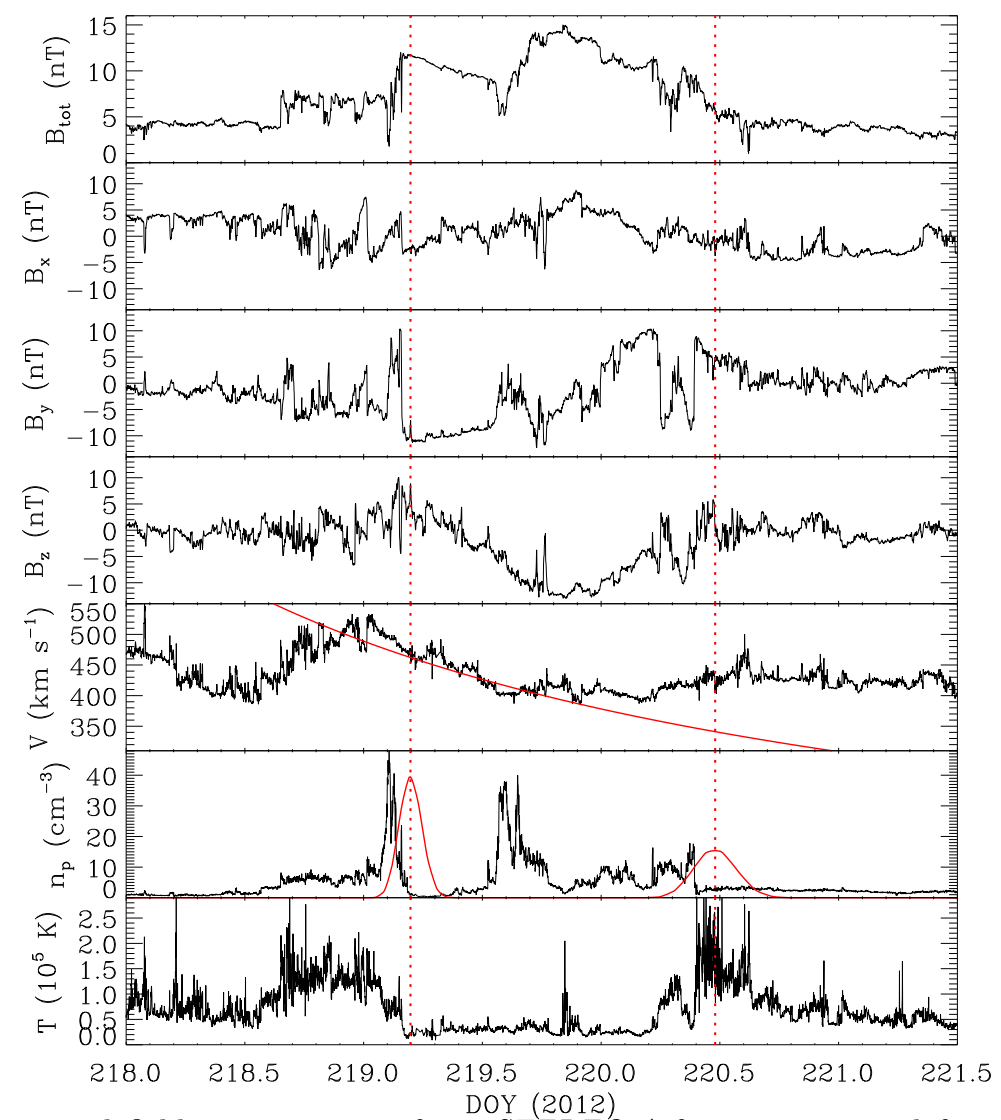

Figure 5. In situ plasma and field measurements from STEREO- $A$ for a time period from 2012 August 5 (DOY=218) to 2012 August 8 (DOY=221). The top panel shows the total magnetic field, with the individual field components shown below it in a spacecraft-centered RTN coordinate system. The bottom three panels are the proton flow speed, number density, and temperature. The solid red lines in the speed and density panels are the values predicted from the CME-2 kinematic and morphological reconstruction in Figures 3-4. The vertical red dotted lines indicate the predicted MFR encounter time, based on the location of the predicted density peaks.

than those actually observed (Wood et al. 2017), meaning that there is usually a significant mismatch between the size scales of the inferred MFR structure from imaging and in situ data. The mismatch seems to be less pronounced in this instance. All of this is encouraging for our efforts to infer a plausible 3-D field structure for CME-2 that is consistent with both the imaging and in situ data.

The in situ ICME field structures that are considered to provide the strongest cases for the MFR paradigm are those classified as "magnetic clouds" (MCs), which possess field rotations consistent with passage through an MFR with a helical field (Burlaga et al. 1981; Marubashi 1986; Burlaga 1988; Bothmer \& Schwenn 1998). The ICME in Figure 5 does exhibit field rotations, with $B_{y}$ in particular changing from negative to positive, but the field variations are not very smooth, and possess many irregularities. The $B_{t o t}$ profile is irregular, and there is also a large density peak in the middle of the ICME region for which we have no explanation. All of this suggests that the presumed MFR of CME-2 may be disturbed in a way that could make it difficult to model precisely. This might in principle be caused by interactions between CME-1 and CME-2, which overlap a little in Figure 4. This modest overlap develops as CME-2 reaches its final velocity early in the COR2 FOV, and the degree of overlap is not expected to change much at later times due to the similar final speeds of the two CMEs.

Nevertheless, we can still make some strong qualitative inferences about the field geometry of CME-2 from the field characteristics seen in Figure 5 (e.g., Nieves-Chinchilla et al. 2019). The images of CME-2 strongly imply a N-S oriented MFR, as shown in Figure 4. This means that it is the $B_{z}$ panel of Figure 5 that should tell us the direction of the MFR's central axial field, and the data in Figure 5 provide a very clear answer. The $B_{z}$ field in the ICME is negative, meaning that the axial field of the MFR is to the south. The $B_{y}$ field profile then tells us the handedness of the azimuthal field around the central axial field. With $B_{y}$ being initially negative, but changing to generally positive halfway through the ICME period, the clear inference is that the azimuthal field is right-handed.

This inferred MFR field geometry means that the field at the outer surface of the CME-2 MFR should be pointed back toward Earth in Figure 4(b). There is therefore a very clear expectation for the rotation measure for the VLA LOS when it first encounters CME-2. It must be positive. Fortunately, this is exactly what is observed (see Section 4 

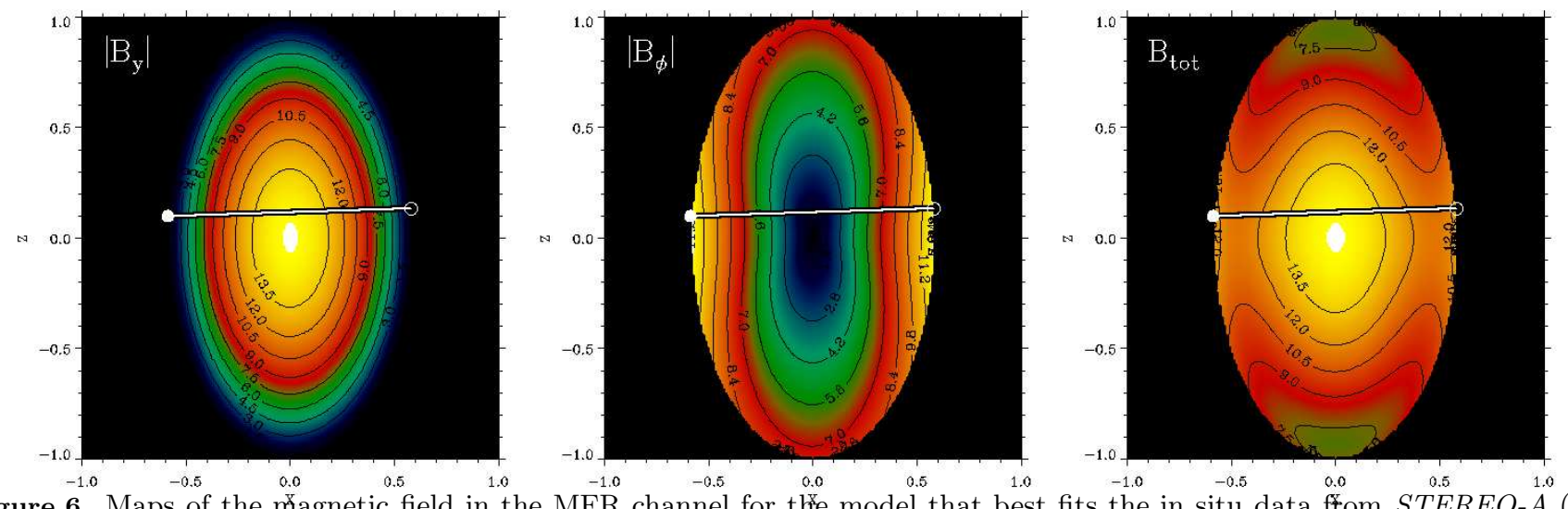

Figure 6. Maps of the magnetic field in the MFR channel for the model that best fits the in situ data from $S T E R E O-A^{-0.5}$ (see Figure 7). From left to right, the maps show the axial field, the azimuthal field along the elliptical contours of the MFR channel, and the total field. The contours indicate the field values in units of $\mathrm{nT}$. The values shown correspond to the field at the apex of the MFR at the time the spacecraft first enters the MFR. The white line indicates STEREO-A's path through the MFR channel, entering from the right and exiting to the left.

and Kooi et al. 2017). If the rotation measure had instead been negative in sign, reconciling the radio data with the CME field structure inferred from the imaging and STEREO-A in situ data would be impossible, which would in turn lead to serious questions about how images and in situ observations of CMEs are generally interpreted in the MFR paradigm. All this illustrates the value of multiple observational constraints on CME structure, to provide definitive tests as to the self-consistency of the observations with any assumed field geometry for the structure.

Moving from purely qualitative inferences of field geometry to more quantitative results requires the use of a physical model for MFR structure. For this purpose, we use the recently developed model of TNC18. Traditional modeling of ICME field structure has generally assumed that an MFR can be approximated locally as an infinite cylinder with a circular cross section, and with a force-free field (e.g., Lepping et al. 1990). Nieves-Chinchilla et al. (2016) presented a modified modeling approach that relaxes the force-free assumption, and this model has now been further expanded to consider elliptical MFR channels, as opposed to purely circular ones (TNC18). This is an important advance for our purposes, as CME-1 and CME-2 are here both inferred to have elliptical MFR channels, with $\eta_{s}=1.5$ and $\eta_{s}=1.7$, respectively, and such ellipticity is by no means uncommon (Wood et al. 2017).

The MFR model of TNC18 is developed in a coordinate system where the MFR is pointed along the y-axis and the flux rope channel is therefore mapped in the xz-plane. (See Figure 1 of TNC18.) In this coordinate system, the field can be reduced to two components, an axial field, $B_{y}$, along the y-axis, and an azimuthal field, $B_{\phi}$, directed around the origin in the xz-plane. The total field is then simply $B_{t o t}=\sqrt{B_{y}^{2}+B_{\phi}^{2}}$. It is crucial to note that in the elliptic-cylindrical coordinate system used by TNC18, the direction of $B_{\phi}$ is not circular about the origin like in a normal polar coordinate system, but is instead along the elliptical contours defined by the ellipticity assumed for the MFR shape, which for our purposes here is based on that inferred from images (e.g., $\eta_{s}=1.7$ for CME-2).

We here quantify the field using two parameters, the axial field at the MFR center, $B_{t}$, and the maximum azimuthal field at the surface of the MFR, $B_{p}$. We now briefly describe how $B_{t}$ and $B_{p}$ relate to the mathematical formalism of TNC18, but we refer the reader to that paper for details. The model of TNC18 uses polynomial expansions to express the axial and azimuthal components of the current density, with polynomial exponents $m$ and $n$, respectively. They also define a quantity, $\tau$, that relates the axial field at the MFR axis to that at the surface; and they use a parameter $\delta$ to quantify the ellipticity of the MFR, which relates to our $\eta_{s}$ parameter as $\delta=1 / \eta_{s}$. In TNC18, the field components for any pair of indices $n, m$ are basically defined by the quantities $B_{n}^{0}$ and $C_{n m}$, where if $f_{c} \equiv(n+1) /\left(\delta^{2}+m+1\right)$, then $B_{t}=\delta \tau B_{n}^{0}$ and $B_{p}=-\delta f_{c} B_{n}^{0} / C_{n m}$. Following the example case explored by TNC18, we here consider only the pair of indices $m=0$ and $n=1$, and we assume $\tau=1$, which corresponds to the case where the axial field simply falls to zero at the MFR surface.

With these assumptions, the TNC18 MFR model for assumed values of $B_{t}=+15.0 \mathrm{nT}$ and $B_{p}=+12.6 \mathrm{nT}$ is shown in Figure 6, which we will show below is a solution that relates to the best fit to the in situ field measurements of CME-2 from STEREO-A. We avoid ambiguities of sign for now by simply showing in the figure absolute values of $B_{y}$ and $B_{\phi}$. The axial field, $B_{y}$, has the peak value of $B_{t}$ at MFR center, which decreases to zero at the MFR surface, as required for the $\tau=1$ assumption. In contrast, $B_{\phi}$ is zero at the center but increases toward the MFR surface, where we again emphasize that the direction of $B_{\phi}$ is along elliptical contours about the origin rather than circular ones. Its maximum, $B_{p}$, is at the surface on the minor axis of the ellipse. The higher azimuthal fields along the minor 
axis compared to the major axis are required by conservation of magnetic flux about the central axis.

Our 3-D reconstructed MFR shapes in Figure 4 have cross-sections that vary along the MFR. In particular, the cross-sectional area is large near the apex and smaller in the legs. A spacecraft moving through the MFR can in principle pass through parts of the MFR with different cross-sectional areas, which we must take into account in modeling the field components that the spacecraft sees. The TNC18 MFR model does not by itself tell us how to do this. However, a 3-D MFR model must conserve magnetic flux, both axially and azimuthally, and we use these flux conservation properties to guide us in relating fields in one part of the MFR to those in another part.

We first need to define a reference cross section for the MFR, and we naturally choose the apex. At the apex, we use $a_{m i n}^{a p}$ to represent the minor radius of the MFR, with field components $B_{y}^{a p}$ and $B_{\phi}^{a p}$. The question then is, what are the field components at a different part of the MFR with a different (presumably lower) minor radius, $a_{\min }$ ? For the axial field, the integrated magnetic flux will be proportional to the cross-sectional area of the MFR, which will be proportional to $a_{m i n}^{2}$, so at an arbitrary part of the MFR, $B_{y}=B_{y}^{a p}\left(a_{m i n}^{a p} / a_{m i n}\right)^{2}$. For the azimuthal field, the integrated magnetic flux for a distance element $d s$ along the MFR is proportional to $a_{\min } B_{\phi} \cdot d s$. Assuming the same azimuthal flux for each distance element along the MFR therefore requires $B_{\phi}=B_{\phi}^{a p}\left(a_{m i n}^{a p} / a_{m i n}\right)$. With this assumption, note that both $B_{y}$ and $B_{\phi}$ increase as you move down the legs of the MFR, where the cross-sectional area is lower, but $B_{y} \propto 1 / a_{\text {min }}^{2}$ increases more than $B_{\phi} \propto 1 / a_{m i n}$, meaning that the overall MFR field becomes more axial in the legs than near the apex.

Another complication is that the MFR is not static as the spacecraft moves through it. It is after all the CME's radial motion away from the Sun that is mostly moving the spacecraft through the MFR structure, and not the spacecraft's heliocentric motion. The MFR will be expanding during the spacecraft encounter, and this expansion of the MFR must also be taken into account. We have simply assumed self-similar expansion in the 3-D reconstruction process described in Section 3. The defining characteristic of self-similar expansion is that all distances scale the same in order to preserve the shape of the expanding structure. The expansion as a function of time is described by the kinematic model shown in Figure 3(b), which provides the leading edge distance of the CME, $R_{l e}$, as a useful reference distance. Both $a_{\min }$ and the overall length of the MFR, $L$, will scale linearly with $R_{l e}$. Magnetic flux conservation requires $B_{y} \propto 1 / a_{\min }^{2}$ and $B_{\phi} \propto 1 /\left(a_{\min } L\right)$, so both $B_{y}$ and $B_{\phi}$ will be proportional to $1 / R_{l e}^{2}$. Both components of the magnetic field will therefore decrease with time in the same manner as the MFR expands.

The preceding two paragraphs describe how we treat the spatial and time dependence of the field within the 3-D MFR shapes inferred from the imaging. With this established, it is now possible for us to take a cross-sectional field map like that in Figure 6 for the apex of the MFR at one time, and extrapolate from it the field structure of the full 3-D MFR not only at that reference time, but to any other time as well based on the kinematic model from Figure 3(b). We can now proceed to fit the in situ field observations of CME-2 from STEREO-A to find a best-fit field structure in the context of the TNC18 model.

A first step is simply to identify the track of the spacecraft through the MFR, which in Figure 4 is represented as a blue arrow. White lines in Figure 6 show the STEREO-A track through the MFR channel, which moves from right to left. This is a near direct hit on the spacecraft, with STEREO-A passing very close to the axis of the MFR. There is a slight positive slope to the track, which is due to the expansion of the MFR channel during STEREO-A's passage through it, which in a relative sense moves the spacecraft closer to the MFR's minor axis.

Figure 7 reproduces the the STEREO-A magnetic field measurements shown in Figure 5. The goal is to fit these data using the TNC18 MFR model, operating in the MFR geometry inferred from the image analysis, with $B_{t}$ and $B_{p}$ being the two free parameters. In doing this, we have to make the coordinate transformations from the TNC18 coordinate system used in Figure 6 to STEREO-A's RTN coordinate system, which defines the $B_{x}, B_{y}$, and $B_{z}$ field components in Figures 5 and 7 . The best fit to the data is found using $\chi^{2}$ minimization (Bevington \& Robinson 1992). We not only fit $B_{x}, B_{y}$, and $B_{z}$ simultaneously, but also $B_{t o t}$. Including $B_{t o t}$ seems redundant, but we see value in considering the positive-definite $B_{t o t}$ quantity in the fit. Uncertainties for the $B_{t o t}, B_{x}, B_{y}$, and $B_{z}$ data points must be estimated to compute the $\chi^{2}$ quality-of-fit factor. For $B_{t o t}$, we divide the mean $B_{t o t}$ within the MFR encounter time by 30 and take that as our uncertainty estimate. For the individual components, we compute the mean absolute value of $B_{x}, B_{y}$, and $B_{z}$ within the encounter time and divide that by 10 to represent the uncertainty estimate. The field variations within the MFR channel are uneven, and exhibit anomalies that the MFR model cannot hope to fit, such as the big double-dip decrease in $B_{y}$ near the end of the MFR encounter, but we here have made no effort to remove such anomalies before fitting the data.

There are only two free parameters of the fit, $B_{t}$ and $B_{p}$. The best fit to the data is shown in Figure 7, with values of $B_{t}=+15.0 \mathrm{nT}$ and $B_{p}=+12.6 \mathrm{nT}$. As emphasized above, these values have to be defined for a specific place and time within the MFR. The quoted values are for the apex of the MFR at the time when the MFR first reaches STEREO-A. 


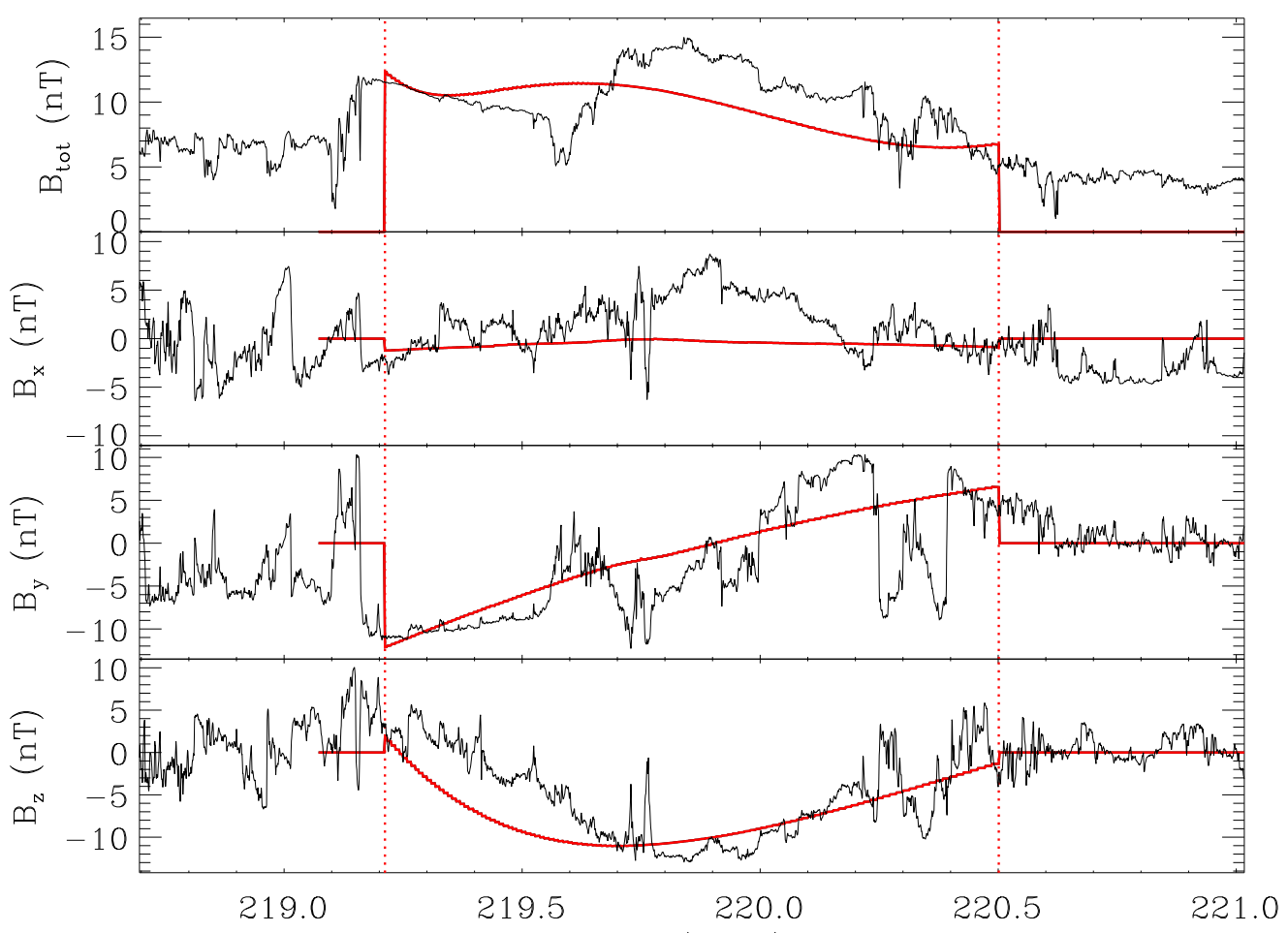

Figure 7. The STEREO-A magnetic field measurements from Figure 5 are reproduced. The red lines indicate a best-fit to the field values. The two free parameters of the fit are the central axial field $\left(B_{t}=+15.0 \mathrm{nT}\right)$ and the maximum azimuthal field at the MFR surface $\left(B_{p}=+12.6 \mathrm{nT}\right)$.

The field maps in Figure 6 correspond to this particular best-fit field model. The field values at the actual location where the spacecraft hits the MFR will be somewhat different, although in this particular case the difference is tiny. The general decrease in model $B_{t o t}$ in Figure 7 is due to the expansion of the MFR during the spacecraft encounter time, which decreases the field with time. As a sign convention, we take the polarity of the western leg of the MFR to be the sign of $B_{t}$. For CME-2, which is oriented close to N-S, the northern leg is slightly west of the southern one, so a positive $B_{t}=+15.0 \mathrm{nT}$ result means that the northern leg is positive and the southern one negative, leading to the negative $B_{z}$ seen in the in situ data. For $B_{p}$, positive (negative) values indicate a right-handed (left-handed) azimuthal field. Thus, our $B_{p}=+12.6 \mathrm{nT}$ result indicates a right-handed orientation for the azimuthal field, leading to the rotation of $B_{y}$ from negative to positive values in Figure 7 .

In analyses of this nature, we imagine that it could often be necessary to introduce a couple extra free parameters into the fit to make the MFR arrival and encounter times at the spacecraft agree better with the in situ data. However, as described above, for this particular case our MFR reconstruction has ended up with a predicted arrival time and event duration that already look very plausible, so no additional scaling parameters are deemed necessary. We consider the quality of the fit to the data in Figure 7 to be impressive considering that there are only two free parameters. In most past published fits of this nature, there are far more free parameters (e.g., Lepping et al. 2011, 2015), because there are many parameters associated with the geometry of the MFR and the spacecraft's path through it. In our analysis, the geometry and path are entirely fixed by the image-based 3-D MFR reconstruction, leaving only the field parameters to vary.

\section{COMPARISON WITH RADIO FARADAY ROTATION OBSERVATIONS}

We now turn our attention to modeling the radio Faraday rotation observations for the two lines of sight occulted by the 2012 August 2 CMEs. The VLA observations are described in detail by Kooi et al. (2017). The two background polarized radio sources are both radio galaxies: Source 0842 (full name $0842+1835$ ) occulted by CME-2, and source 0843 (full name 0843+1547) occulted by CME-1. These lines of sight are shown as red and orange lines in Figure 4, respectively. Both lines of sight pass within about $10 \mathrm{R}_{\odot}$ from Sun-center at closest approach, within the LASCO/C3 FOV. Kooi et al. (2017) interpreted the LASCO/C3 images to suggest that the 0843 LOS grazes the southern edge of CME-2, and is thereby occulted by both CMEs, although CME-1 clearly dominates the RM signal. Our reconstruction here has CME-2 narrowly missing the 0843 LOS, so we will here be intepreting the 0843 data in the context of CME- 1 occultation only. 


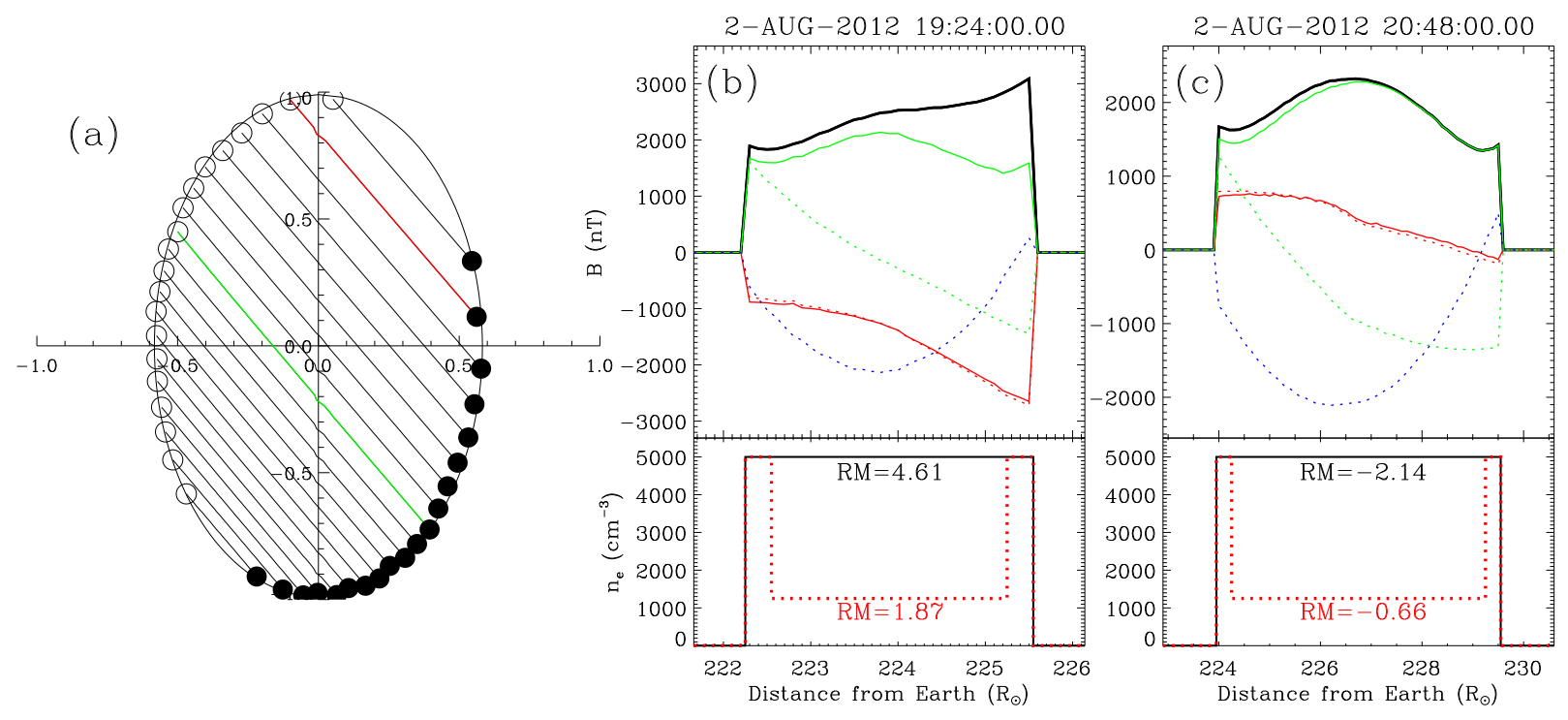

Figure 8. (a) Traces of the LOS to source 0842 through the model MFR channel of CME-2 as a function of time, for 12-minute increments starting at UT 19:12 on 2012 August 2, with the LOS from Earth entering from the top left (open circles) and exiting toward the bottom right (filled circles), based on the 3-D reconstruction in Figure 4. (b) The red, green, and blue dotted lines are traces of $B_{x}, B_{y}$, and $B_{z}$, respectively, in GSE coordinates for the red track through the MFR channel in (a) based on the MFR reconstruction inferred by fitting STEREO-A in situ data. The red and green solid lines are the projections along the LOS, $B_{\|}$and $B_{\perp}$, respectively. The black line is the total field, $B_{t o t}$. The lower panel shows two assumed electron density profiles, and the RM observations that result from them (in $\mathrm{rad} \mathrm{m}^{-2}$ units). (c) Analogous to (b), but for the green track in panel (a).

The Faraday rotation diagnostic relies on detecting the change in polarization position angle ( $\chi$; defined by the electric field vector) induced by the passage of a CME in front of the background source. This rotation is

$$
\Delta \chi=\left[\left(\frac{e^{3}}{2 \pi m_{e}^{2} c^{4}}\right) \int_{L O S} n_{e} \mathbf{B} \cdot \mathbf{d s}\right] \lambda^{2}=[R M] \lambda^{2},
$$

where $\lambda$ is the observed radio wavelength, and $\mathbf{d s}$ is the differential direction vector along the LOS. The term in square brackets is the rotation measure (RM), which represents the quantity of interest, with units of $\mathrm{rad} \mathrm{m}^{-2}$. The constant within the parentheses includes the electron charge $(e)$, the electron mass $\left(m_{e}\right)$, and the speed of light $(c)$. The rotation measure is the integral of the parallel component of the field times the electron density along the LOS, multiplied by the constant in parentheses in Equation (2). Thus, modeling RM requires both a field model for the CME and assumptions about the density distribution within it.

\subsection{Analysis of Source 0842 and CME-2}

We first discuss the source 0842 observations, which provide RM diagnostics for CME-2. The STEREO-A observations of CME-2 have allowed us to model the field of this CME based on the in situ data alone, in the context of the TNC18 MFR model and the CME-2 MFR shape inferred from the images (see Section 3). Thus, with the CME field structure already fixed, the only degrees of freedom we have for modeling RM for source 0842 are those associated with the CME density.

The first step in modeling RM is to determine the track of the observed LOS through the CME as a function of time. We can do this thanks to the full 3-D reconstruction of the MFR outline provided by the morphological analysis described in Section 2. Results are shown in Figure 8(a). The figure shows schematically the path of the LOS to source 0842 through the MFR channel as a function of time, in 12-minute increments. The LOS from Earth enters the MFR on the top left and and then traverses the MFR channel toward the bottom right. The paths move downwards relative to the MFR as a function of time, passing through the center of the MFR at about UT 20:24. The LOS trajectory is also illustrated in Figure 4(b), which shows the LOS entering the MFR on the side of the channel and exiting near the leading edge of the CME, consistent with the early paths in Figure 8(a).

With the tracks through the MFR established, we can then use the time-dependent, 3-D MFR model described in Section 3 to trace the field components along the LOS as a function of time. This is shown in Figure 8(b-c) for two example time steps. The dotted lines in the upper panels indicate $B_{x}, B_{y}$, and $B_{z}$. These components are provided in 


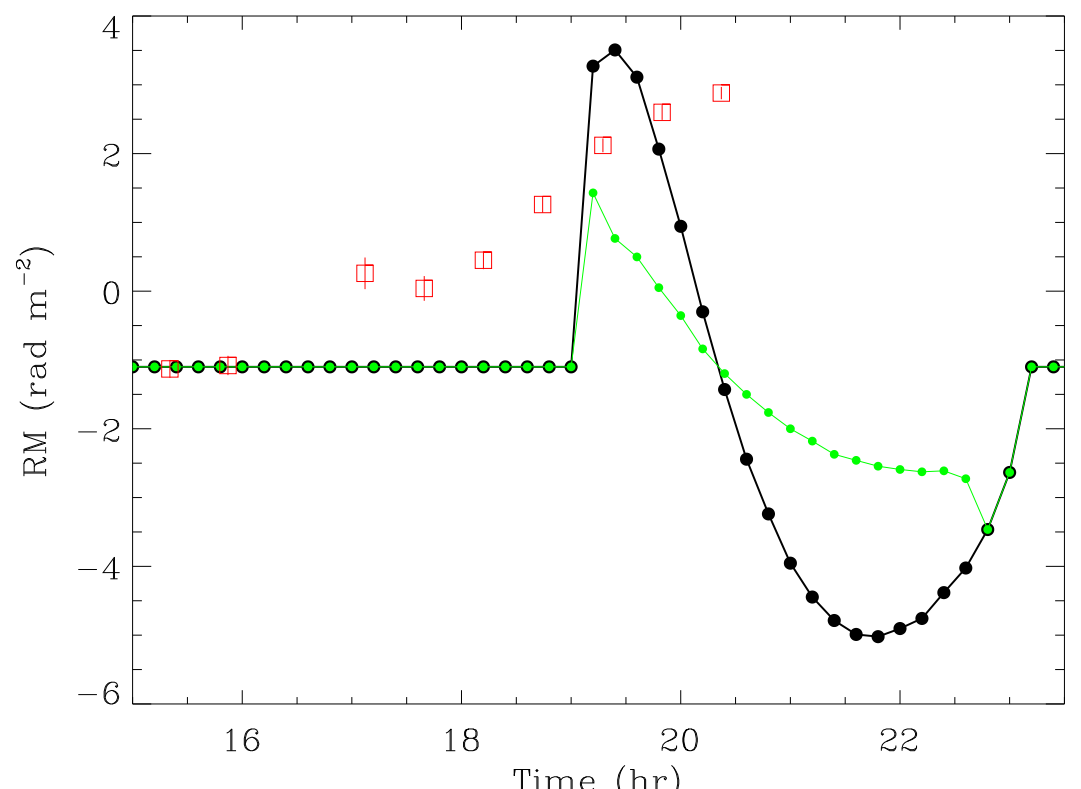

Figure 9. The red data points are VLA observations of RM for source 0842 from Kooi et al. (2017). The black and green lines are predicted RM values using the reconstructed MFR structure based on the imaging and STEREO-A in situ data, and the two electron density profiles in Figure $8(\mathrm{~b}-\mathrm{c})$, with the black line assuming a constant density in the MFR channel, and the green line assuming density peaks at the surface.

a geocentric-solar-ecliptic (GSE) coordinate system, with Earth at the origin, the x-axis pointed at the Sun, the z-axis toward ecliptic north, and the y-axis to the right as viewed from Earth to form an orthogonal system. This seems a natural coordinate system to use given the Earth-based location of the VLA observations.

For RM computation purposes, we need to know the field direction relative to the LOS, so Figure 8(b-c) also shows the $B_{\|}$and $B_{\perp}$ components of the field relative to the LOS, with $B_{\|}$obviously being the quantity of particular interest. Neither $B_{\|}$nor $B_{\perp}$ are really signed quantities, but in the figure we give $B_{\|}$a sign consistent with $B_{x}$ to emphasize that the two are nearly identical in magnitude. This is because the LOS to the background sources that VLA is monitoring are naturally very close the Sun, and therefore they are pointed roughly along the GSE x-axis toward the Sun.

The second quantity needed to compute model RM values, after $B_{\|}$, is the electron density, $n_{e}$. Unlike $B_{\|}$, which is entirely fixed by the Section 3 analysis, $n_{e}$ is unconstrained. In the bottom panels of Figure 8(b-c) there are two different assumed density models. Both density models assume a maximum value of $n_{e}=5000 \mathrm{~cm}^{-3}$. This value is roughly consistent with a $1 / r^{2}$ extrapolation of the $n_{p}$ densities seen by STEREO-A at 1 au back to the $\sim 12 \mathrm{R}_{\odot}$ distance where the 0842 LOS is encountering the CME. One density model (the black line) simply assumes this as a constant density throughout the MFR. The other density model, which we call the shell model (the red dotted line), assumes the peak density is only at the surface of the MFR, with densities a factor of 4 lower in the interior. The RM values computed for the two sample LOS tracks are provided explicitly in the lower panels of Figure 8(b-c) for both density models. In computing these values, it is crucial to note that in radio astronomy the convention is for RM to be positive for fields directed along the LOS toward Earth. This is opposite from the $B_{\|}$(and $B_{x}$ ) sign in the upper panels of Figure 8(b-c), which is associated with the GSE coordinate system instead of the radio convention. Thus, the sign of RM is reversed from that suggested by $B_{\|}$in the figure.

Figure 9 shows the model RM values as a function of time for both the constant density model and the shell model, and compares them with the RM observations provided by VLA. As noted above, refer to Kooi et al. (2017) for details of the VLA observations. The VLA observations suggest that even outside the CME encounter there is a background $\mathrm{RM}$ value of about $-1.1 \mathrm{rad} \mathrm{m}^{-2}$. This is to be expected, as the quiescent solar wind itself will produce an RM signal. Our model RM values are displayed after adding to it this $-1.1 \mathrm{rad} \mathrm{m}^{-2}$ background value.

Our density and field MFR parameters can be compared with those estimated by Kooi et al. (2017). Our $n_{e}=$ $5000 \mathrm{~cm}^{-3}$ value is close to the value of $n_{e}=6900 \pm 500 \mathrm{~cm}^{-3}$ estimated by Kooi et al. (2017). Comparing the field values is somewhat trickier. Our $B_{t}=+15.0 \mathrm{nT}$ and $B_{p}=+12.6 \mathrm{nT}$ values correspond to a time when the MFR edge reaches STEREO-A, at which time the center of the MFR is at about 0.8 au. Extrapolating back to a time when the MFR center is at the $\sim 12 \mathrm{R}_{\odot}$ encounter distance of the $0842 \mathrm{LOS}$ yields values of $B_{t}=+31 \mathrm{mG}$ and $B_{p}=+26 \mathrm{mG}$, which are roughly compatible with the $B_{C M E}=10.4 \pm 0.4 \mathrm{mG}$ value from Kooi et al. (2017), albeit somewhat higher. 
(For reference, note that $1 \mathrm{nT}$ equals $0.01 \mathrm{mG}$.)

In comparing the model and observed RM values for CME-2 in Figure 9, we first emphasize the principle success of the model, namely that the model RM values are positive, consistent with the VLA observations. If the observed RM values had been negative, there would have been no way whatsoever to reconcile the MFR model constructed from our interpretation of the images and STEREO-A in situ data with the VLA data. This would have called into serious question the standard methodologies by which we and others interpret CME images and 1 au in situ data. The successful prediction of positive RM values is therefore of significant importance. The model prediction of positive RM values by VLA is truly a falsifiable prediction for the VLA data, and the model passes this crucial test.

However, there are two worrisome inconsistencies between the model and observed RM values. One is that the observed RM values actually increase about two hours before the MFR reconstruction suggests that CME-2 occults the LOS. Inspection of Figure 8 from Kooi et al. (2017) shows that the LASCO/C3 brightness values at the source 0842 location do not really start increasing until after UT 18:30, so it is not surprising that our image-based MFR reconstruction does not predict any RM signal until after this time. We here interpret the premature RM increase as being due to a sheath region of deflected solar wind field out ahead of the CME-2 MFR.

The STEREO-A in situ data in Figure 5 actually suggest the existence of just such a precursor sheath region, between $\mathrm{DOY}=218.6$ and $\mathrm{DOY}=219.0$. This time range is ahead of the actual time period of the ICME encounter, which presumably begins with the big density peak at DOY $=219.1$, where the low $T$ and high $B_{\text {tot }}$ values truly start. There is clearly a region of disturbed $B$ and slightly enhanced $n_{p}$ ahead of this time. For a relatively slow CME like CME-2, there is not really any shock associated with the CME, but there will still be a region of disturbed solar wind and deflected $B$ out ahead of the CME, which could affect the RM values observed by VLA even before the CME actually occults the LOS. [See, e.g., Kilpua et al. (2017) for a discussion of ICME shock sheaths and sheath-like regions.] This is therefore our interpretation for why the observed RM values in Figure 9 increase relative to the $\mathrm{RM}=-1.1 \mathrm{rad} \mathrm{m}^{-2}$ background even before the CME arrival. However, if a sheath region is affecting the RM values, this is potentially a significant source of uncertainty regarding the interpretation of the RM values that are observed even after the CME actually occults the LOS, given that the sheath of deflected ambient solar wind field could be affecting those values as well.

The second worrisome inconsistency between the model and observed RM values in Figure 9 is that the model clearly requires that the sign of RM change from positive to negative relatively quickly. Note that both the constant density and shell model RM predictions suggest a change in RM sign at about UT 20:20, roughly when the LOS passes through the center of the MFR channel (see Figure 8(a)). No change in the assumed density model will affect this prediction significantly, as it is determined almost entirely by the field model, which is constrained by the $S T E R E O-A$ in situ data. In contrast, the actual VLA observations do not show any indication that the positive RM values reverse sign. Unfortunately, the VLA data end too early, the last VLA data point being at UT 20:20. This makes it very hard for us to assess the seriousness of the discrepancy. Even just one more hour of VLA data would have been very illuminating. If that hour had shown the beginnings of an RM sign reversal, we could have concluded that this is consistent with the model and that the delayed reversal is simply due to inaccuracies in the field model resulting from the fit to the in situ data in Figure 7. However, if that extra hour had shown no evidence for any reversal whatsoever, we would have concluded that this is a serious inconsistency with the single MFR reconstruction of CME-2, and that our interpretation of the images and STEREO-A in situ data for this event is therefore in serious doubt as a consequence. In the absence of this extra hour of VLA data, we instead have to consider the issue as still being ambiguous.

Finally, it should be noted that the shell model for the CME densities is clearly not favored by the data in Figure 9 . The shell model predicts rather sharp maxima in density when the CME first occults the LOS, which are not observed. This is also the case for CME-1, although we will not demonstrate that explicitly in the next section. For CME-1 we will exclusively assume a constant density model. This provides evidence that the shell model is a worse approximation for these CMEs than the constant density model.

\subsection{Analysis of Source 0843 and CME-1}

We now turn our attention to the source 0843 data, and CME-1. Figure 10(a) shows the paths of the LOS through the model CME-1 MFR channel, in 12-minute increments starting at UT 16:48 on 2012 August 2 and extending for 9.2 hours thereafter. Figure 4(a) provides another illustration of how CME-1 is encountering the LOS. The eastern leg of the CME is tilted north of the LOS and therefore the VLA observations of 0843 basically end up probing the western half of the CME, with the LOS at later times moving down the western leg of the MFR as the CME expands outwards. The expansion eventually moves the LOS through the center of the MFR channel, at about UT 21:24.

In contrast to the CME-2 analysis, there is no 1 au in situ data to constrain the MFR model. Thus, there are 


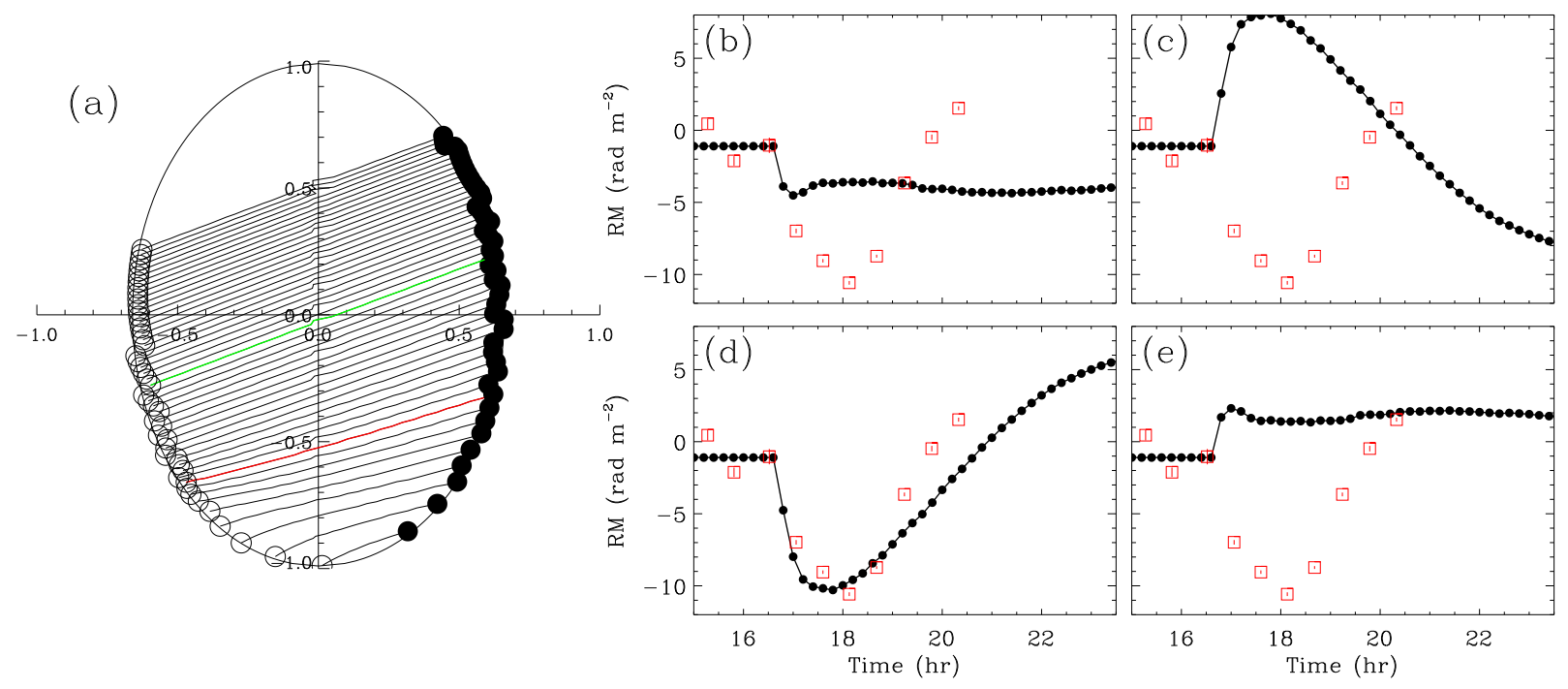

Figure 10. (a) Traces of the LOS to source 0843 through the model MFR channel of CME-1 as a function of time, for 12 -minute increments starting at UT 16:48 on 2012 August 2, with the LOS from Earth entering from the left and exiting to the right, based on the 3-D reconstruction in Figure 4. The red and green lines are paths referred to in Figure 11. (b-e) The red data points show the VLA RM values of source 0843 from Kooi et al. (2017). The black lines are model RM values assuming the following magnetic field component values: (b) $\left(B_{t}, B_{p}\right)=(+10,+10) \mathrm{nT},(\mathrm{c})\left(B_{t}, B_{p}\right)=(+10,-10) \mathrm{nT},(\mathrm{d})\left(B_{t}, B_{p}\right)=(-10,+10) \mathrm{nT}$, and (e) $\left(B_{t}, B_{p}\right)=(-10,-10) \mathrm{nT}$. The data clearly favor the field polarity assumed in (d).

more free parameters to consider when trying to reproduce the VLA RM observations. However, the source 0843 RM constraints on CME-1 shown in Figure 10(b-e) are, by themselves, better than those for CME-2 in two respects. One is that the RM values are simply larger, and the other is that the sign of RM is observed to reverse at about UT 19:30, which is very helpful for constraining the field model of the MFR even in the absence of 1 au in situ constraints.

In Figure 10(b-e), we show the RM values predicted by an MFR model assuming four possible polarities for the MFR field; in particular with $\left(B_{t}, B_{p}\right)=(+10,+10) \mathrm{nT},\left(B_{t}, B_{p}\right)=(+10,-10) \mathrm{nT},\left(B_{t}, B_{p}\right)=(-10,+10) \mathrm{nT}$, and $\left(B_{t}, B_{p}\right)=(-10,-$ 10) $\mathrm{nT}$. Analogous to what we did for the CME-2 analysis, these values are for the apex of the MFR, for the time when the leading edge of the MFR first reaches $1 \mathrm{au}$, and we are once again assuming a background RM value of -1.1 $\mathrm{rad} \mathrm{m}^{-2}$. We are using the 1 au arrival time as the reference time, even in the absence of any relevant data at that time for this CME, in order to allow for easier comparison with the $\left(B_{t}, B_{p}\right)=(+15.0,+12.6) \mathrm{nT}$ best-fit values found for CME-2 (see Figure 7). As for the density model, in Figure 10(b-e) we simply assume a constant density within the MFR channel of $n_{e}=10,000 \mathrm{~cm}^{-3}$, a value about a factor of two lower than estimated by Kooi et al. (2017).

The $(-,+)$ polarity model in Figure 10(d) is by far the favored polarity. The $(+,-)$ and $(-,-)$ polarities of panels (c) and (e) are clearly wrong, given that they yield initial positive RM values instead of the observed negative values. The $(+,+)$ polarity of panel (b) does yield negative RM values, but the model shows no hint of reversing the RM sign at later times, in contrast with the data. Thus, the panel (d) model is clearly best, with the $\left(B_{t}, B_{p}\right)=(-10,+10) \mathrm{nT}$ field model combined with the constant-density $n_{e}=10,000 \mathrm{~cm}^{-3}$ model yielding a plausible match to the data. It should be emphasized that in the absence of 1 au constraints, there is a degeneracy between the field and density values. If we arbitrarily increased the field by a factor of 2 and decreased the density by a factor of two, the model RM predictions would be the same, for example.

In Figure 11, we present a model that provides a somewhat better fit to the observations than Figure 10(d), with the RM sign change pushed to an earlier time. This model assumes $\left(B_{t}, B_{p}\right)=(-20,+5) \mathrm{nT}$, with the densities remaining at $n_{e}=10,000 \mathrm{~cm}^{-3}$. Figure 11(a-b) are analogous to Figure 8(b-c), explicitly showing the field components of this best-fit model for two example time steps. The second time step is at the time the LOS crosses the center of the MFR, by which time the sign of RM has already reversed from its initial sign. Figure 11 shows the fit to the data resulting from this model, which is a much better fit than we found for the CME-2 data. However, the better quality fit is in part allowed by the freedom we have to assume field model parameters to be anything we want, in absence of any $1 \mathrm{au}$ in situ constraints. Nevertheless, the model is clearly more successful in reproducing the arrival time of the CME at the LOS. There is no precursor RM signature like we found for in the CME-2 analysis, which we interpreted as indicative of an RM signature of a sheath region. 

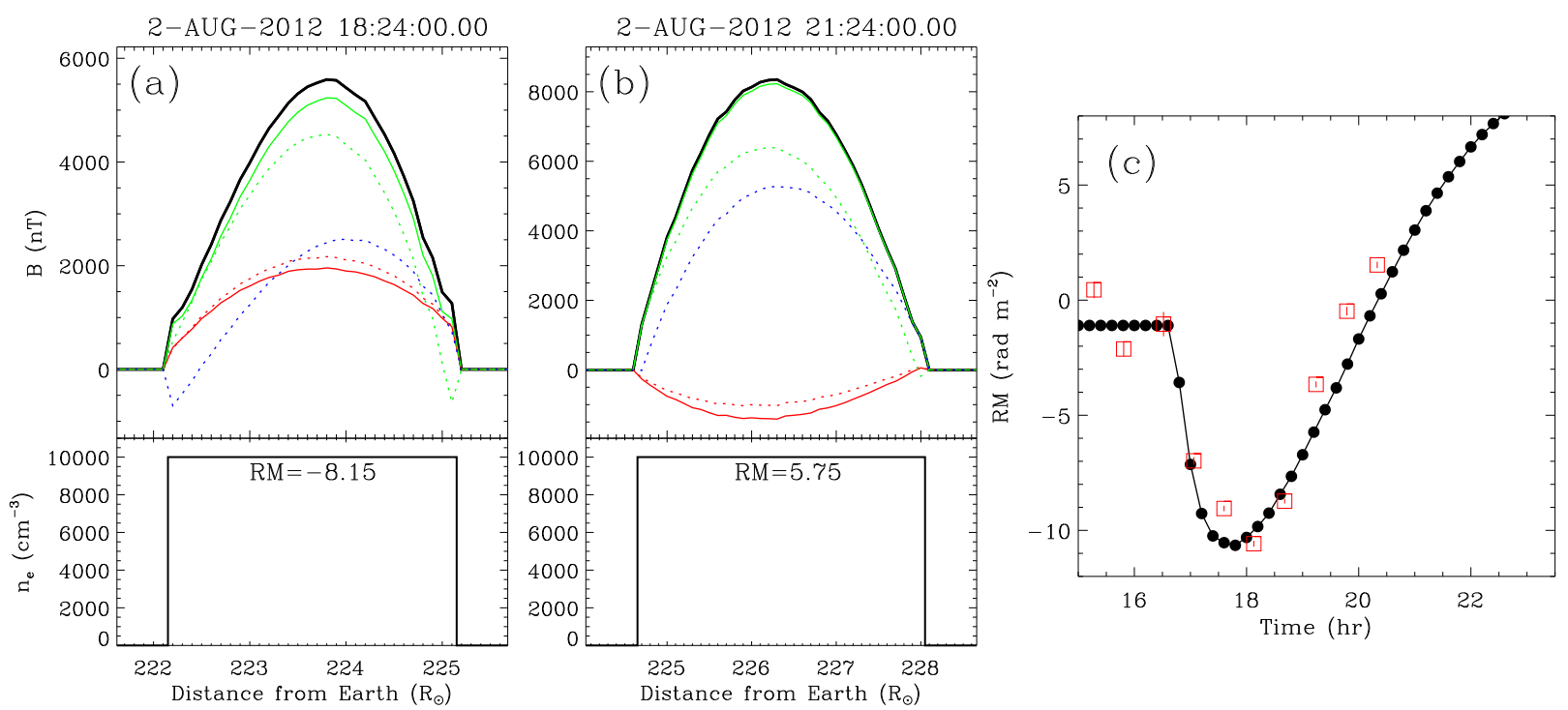

Figure 11. (a) The red, green, and blue dotted lines are traces of $B_{x}, B_{y}$, and $B_{z}$, respectively, in GSE coordinates for the red track through the model CME-1 MFR channel from Figure $10(\mathrm{a})$, assuming a field model with $\left(B_{t}, B_{p}\right)=(-20,+5) \mathrm{nT}$. The red and green solid lines are the projections along the LOS, $B_{\|}$and $B_{\perp}$, respectively. The black line is the total field, $B_{t o t}$. The lower panel shows the electron density profile assumed to compute the displayed RM value (in rad $\mathrm{m}^{-2} \mathrm{units}$ ). (b) Analogous to (a), but for the green track in Figure 10(a). (c) The model RM values for source 0843 occulted by CME- 1 as a function of time (black line), compared with the VLA observations (red boxes).

The $(-,+)$ polarity of the CME-1 MFR implies that the central axial field of the roughly E-W oriented MFR is directed from east to west, and the azimuthal field about the axis is right-handed. The western leg of CME- 1 and the southern leg of CME-2 partly overlap in Figure 4. These are both negative polarity legs in our MFR reconstructions.

\section{SUMMARY}

We have studied the magnetic field structures of two CMEs (CME-1 and CME-2) that erupt from the west limb as viewed from Earth on 2012 August 2, for which there are uniquely extensive observational constraints. These constraints include excellent stereoscopic imaging observations from STEREO and SOHO/LASCO, allowing reconstruction of the 3-D MFR structure of the two events. Both CMEs also occult two separate lines of sight observed by VLA (sources 0843 and 0842 , respectively), which provide constraints on the field strength and orientation of the two CMEs. Finally, CME-2 hits STEREO-A on August 6, making this CME particulary notable as being the first CME with constraints from stereoscopic imaging, 1 au in situ data, and radio RM observations. Our analysis considers all of these data, in the context of a physical MFR model from TNC18. Our findings are summarized as follows:

1.: For CME-2, the combination of imaging and STEREO-A in situ data allows a full time-dependent 3-D model of the MFR field structure to be constructed. This best-fit model has a central axial field, $B_{t}$, and maximum surface azimuthal field, $B_{p}$, of $\left(B_{t}, B_{p}\right)=(+15.0,+12.6) \mathrm{nT}$. These values are quoted for the MFR apex at a time when the leading edge of that apex reaches STEREO-A. A density model simply assuming a constant density within the MFR channel, with $n_{e}=5000 \mathrm{~cm}^{-3}$, seems to work better for reproducing the RM measurements than a shell model with peak densities at the surface of the CME.

2.: The CME-2 MFR reconstruction makes two clear falsifiable predictions for the radio RM observations: 1 . The sign of RM observed by VLA toward source 0842 must be positive, and 2. The sign of RM should reverse to negative within a couple hours. The VLA data pass the first test. This is significant, as a negative result would have invalidated the single MFR model of the CME, potentially casting doubt on the kinds of assumptions that are often made when interpreting images of CMEs and 1 au in situ data. However, the quality of the overall fit to the observed RM values is not great. In particular, the VLA RM observations do not seem to pass the second test, showing no indication of a sign reversal, but unfortunately the data end too early to be sure of the significance of this discrepancy.

3.: For CME-2, the RM values actually increase a couple hours prior to when the MFR reconstruction predicts that the CME occults the 0842 LOS. We interpret this as being due to a sheath region of deflected field out ahead 
of the MFR that is itself producing an RM signature. The existence of RM signatures from CME sheaths could significantly complicate interpretations of radio Faraday rotation from CMEs.

4.: Although there are no 1 au in situ constraints for CME-1, the radio data by themselves provide strong constraints on the field geometry of the CME, since the RM observations show a sign reversal, the timing of which has substantial diagnostic power. Our best-fit model has $\left(B_{t}, B_{p}\right)=(-20,+5) \mathrm{nT}$, where we here quote the values extrapolated to $1 \mathrm{au}$ to allow for ease of comparison with the CME-2 fit. With a simple assumption of constant density throughout the MFR, the best-fit model has $n_{e}=10,000 \mathrm{~cm}^{-3}$.

5.: The model MFR density and field values of CME-1 and CME-2 reported here are within roughly a factor of $2-3$ of those inferred in the previous analysis of Kooi et al. (2017), an acceptable level of agreement considering the different underlying assumptions of these independent analyses.

This study reveals the promise of joint radio, white light imaging, and in situ studies of CMEs, although obtaining more such observations in future observing campaigns will rely on good fortune, given the unpredictable nature of solar transients. Further progress could also be achieved by the monitoring of a larger number of background radio sources behind a CME, allowing for the development of a more detailed model of 3-D magnetic field structure, even in the absence of in situ field measurements from an encounter with a spacecraft.

Financial support was provided by the Chief of Naval Research, and by NASA award 80HQTR18T0084 to the Naval Research Laboratory. The STEREO/SECCHI data are produced by a consortium of NRL (US), LMSAL (US), NASA/GSFC (US), RAL (UK), UBHAM (UK), MPS (Germany), CSL (Belgium), IOTA (France), and IAS (France). In addition to funding by NASA, NRL also received support from the USAF Space Test Program and ONR. This work has also made use of data provided by the STEREO PLASTIC and IMPACT teams, supported by NASA contracts NAS5-00132 and NAS5-00133.

\section{REFERENCES}

Acuña, M. H., Curtis, D., Scheifele, J. L., et al. 2008, Space Sci. Rev., 136, 203

Al-Haddad, N., Nieves-Chinchilla, T., Savani, N. P., et al. 2013, Sol. Phys., 284, 129

Bevington, P. R., \& Robinson, D. K. 1992, Data Reduction and Error Analysis for the Physical Sciences (New York: McGraw-Hill)

Billings, D. E. 1966. A Guide to the Solar Corona (New York: Academic Press)

Bird, M. K., Schruefer, E., Volland, H., \& Sieber, W. 1980, Nature, 283, 459

Bird, M. K., Volland, H., Howard, R. A., et al. 1985, Sol. Phys., 98, 341

Bisi, M. M., Jensen, E., Sobey, C., et al. 2016, Abstract SH11C-2251 presented at 2016 AGU Fall Meeting

Bothmer, V., \& Schwenn, R. 1998, Ann. Geophys., 16, 1

Brueckner, G. E., Howard, R. A., Koomen, M. J., et al. 1995, Sol. Phys., 162, 357

Burlaga, L. F. 1988, JGR, 93, 7217

Burlaga, L., Sittler, E., Mariani, F., \& Schwenn, R. 1981, JGR, 86,6673

Eyles, C. J., Harrison, R. A., Davis, C. J., et al. 2009, Sol. Phys., 254,387

Galvin, A. B., Kistler, L. M., Popecki, M. A., et al. 2008, Space Sci. Rev., 136, 437

Gibson, S. E., \& Low, B. C. 1998, ApJ, 493, 460

Gopalswamy, N., Yashiro, S., Michalek, G., et al. 2009, EM\&P, 104,295

Howard, R. A., Moses, J. D., Vourlidas, A., et al. 2008, Space Sci. Rev., 136, 67

Howard, T. A., Stovall, K., Dowell, J., Taylor, G. B., \& White, S. M. 2016, ApJ, 831, 208

Hu, Q., \& Sonnerup, B. U. Ö. 2001, GeoRL, 28, 467
Illing, R. M. E., \& Hundhausen, A. J. 1985, JGR, 90, 275

Ingleby, L. D., Spangler, S. R., \& Whiting, C. A. 2007, ApJ, 668, 520

Jensen, E. A., Heiles, C., Wexler, D., et al. 2018, ApJ, 861, 118

Kilpua, E., Koskinen, H. E. J., \& Pulkkinen, T. I. 2017, LRSP, 14,5

Kilpua, E. K. J., Mierla, M., Rodriguez, L., et al. 2012, Sol. Phys., 279, 477

Kooi, J. E., Fischer, P. D., Buffo, J. J., \& Spangler, S. R. 2014, ApJ, 784, 68

Kooi, J. E., Fischer, P. D., Buffo, J. J., \& Spangler, S. R. 2017, Sol. Phys., 292, 56

Lepping, R. P., Jones, J. A., \& Burlaga, L. F. 1990, JGR, 95, 11957

Lepping, R. P., Wu, C. -C., Berdichevsky, D. B., \& Szabo, A. 2011, Sol. Phys., 274, 345

Lepping, R. P., Wu, C. -C., Berdichevsky, D. B., \& Szabo, A. 2015, Sol. Phys., 290, 2265

Levy, G. S., Sato, T., Seidel, B. L., et al. 1969, Science, 166, 596

Lugaz, N., Vourlidas, A., \& Roussev, I. I. 2009, Ann. Geophys., 27,3479

Luhmann, J. G., Curtis, D. W., Schroeder, P., et al. 2008, Space Sci. Rev., 136, 117

Marubashi, K. 1986, Adv. Space Res., 6, 335

Möstl, C., Amerstorfer, T., Palmerio, E., et al. 2018, Space Weather, 16, 216

Nieves-Chinchilla, T., Jian, L. K., Balmaceda, L., et al. 2019, Sol. Phys., 294, 89

Nieves-Chinchilla, T., Linton, M. G., Hidalgo, M. A., et al. 2016, ApJ, 823, 27

Nieves-Chinchilla, T., Linton, M. G., Hidalgo, M. A., \& Vourlidas, A. 2018, ApJ, 861, 139 [TNC18] 
Ord, S. M., Johnston, S., \& Sarkissian, J. 2007, Sol. Phys., 245, 109

Robbrecht, E., Berghmans, D., \& Van der Linden, R. A. M. 2009, ApJ, 691, 1222

Sakurai, T., \& Spangler, S. R. 1994, ApJ, 434, 773

Thernisien, A. F. R., Howard, R. A., \& Vourlidas, A. 2006, ApJ, 652,763

Thernisien, A., Vourlidas, A., \& Howard, R. A. 2009, Sol. Phys., 256,111

Vandas, M., \& Romashets, E. P. 2003, A\&A, 398, 801
Vourlidas, A., Lynch, B. J., Howard, R. A., Li, Y. 2014, Sol. Phys., 284, 179

Wood, B. E., Hess, P., Howard, R. A., Stenborg, G., \& Wang, Y. -M. 2020, ApJS, 246, 28

Wood, B. E., \& Howard, R. A. 2009, ApJ, 702, 901

Wood, B. E., Howard, R. A., \& Socker, D. G. 2010, ApJ, 715, 1524

Wood, B. E., Wu, C. -C., Lepping, R. P., et al. 2017, ApJS, 229, 29

Yashiro, S., Gopalswamy, N., Michalek, G., et al. 2004, JGR, 109, A07105 